\title{
Anatomic Location is the Best Predictor of Bacterial Species in Postoperative Infections
}

\begin{abstract}
Introduction: Postoperative surgical site infections are the most common complication following dermatologic surgery. The microbiology of these infections as it relates to patient and surgical characteristics is not well discussed.

Methods: A retrospective chart review on all patients with clinical evidence of wound infection and positive wound culture between 2011-2020 was performed at our tertiary care institution. The microbiology and resistance patterns were investigated in association with patient and surgical characteristics.

Results: Anatomic location was most stronly associated with the type of bacteria cultured, and contributed to the associations between specific bacteria and type of repair (linear, flap, graft) as well as suture material. There was no association between the bacteria cultured and whether the patient was immunosuppressed, smoking, anticoagulated, or diabetic. There was no association between the bacteria cultured and suture technique or procedure type (Mohs micrographic surgery vs excision vs biopsy vs electrodessication and curettage)

Conclusions: Understanding the microbiology of postoperative infections can help dermatologic surgeons in determining the best antibiotic regimen for treatment of these infections. Our study shows that anatomic location is the most important determinant of the type of pathogenic bacteria that will be cultured in a postoperative infection, and thus modifications on antibiotic based on the anatomic location of a surgical site.
\end{abstract}

\section{Introduction}

Dermatologic surgery has historically been associated with low rates of intraoperative and postoperative complications, though postoperative surgical site infections (SSI) are the most commonly reported adverse event. Prior studies have shown infection rates of approximately $1-4.25 \%$ depending on anatomic location and type of procedure performed [1].

SSIs are often diagnosed clinically based on signs and symptoms of pain, warmth, edema, erythema and purulence $[2,3]$. Wound cultures are helpful in identifying culprit bacteria and antibiotic susceptibilities, though the interpretation of results is best performed within a patient's clinical context. In dermatologic surgery, Staphylococcus aureus is the most commonly implicated bacteria in wound infections, followed by Pseudomonas Aeruginosa, Proteus Mirabilis, Enterobacter species, and Serratia Marcescens [1-3]. Understanding of commonly implicated bacteria causing postoperative infections can help surgeons in selecting appropriate prophylaxis and treatment options. Because there are no standardized guidelines, the use of antibiotics is increasing and varies widely, even within institutions [5]. Recent reports have validated prior findings.

We previously investigated below the knee infections at our institution [6]. We reported that patients undergoing wide local excision were more likely to develop a lower extremity SSI than patients undergoing MMS. Further, subcuticular sutures and vertical

\section{Journal of}

\section{Clinical \& Investigative} Dermatology

\author{
Chow M, Miller A and Jiang SIB* \\ Department of Dermatology, University of California, USA
}

\section{*Address for Correspondence}

Jiang SIB, Department of Dermatology University of California, San Diego, Mohs and Dermatologic Surgery 8899 University Center Lane Ste 350, San Diego CA 92122; Email: bjiang@ucsd.edu

Submission: 28 June, 2021

Accepted: 30 July, 2021

Published: 08 August, 2021

Copyright: ( $) 2021$ Chow M, et al. This is an open access article distributed under the Creative Commons Attribution License, which permits unrestricted use, distribution, and reproduction in any medium, provided the original work is properly cited.

mattress sutures were associated with reduced infection rates than other suture techniques. Our goal in performing the study was to expand on this prior study, describe the SSIs that we have encountered and treated over the previous 9 years to identify relationships between cultured bacteria and host and clinical factors, as well as understand the bacterial resistance patterns in our geographic location to better help us treat SSIs going forward.

\section{Methods}

The study was approved by the Institutional Review Board of the University of California, San Diego. Using the electronic medical record, the authors reviewed records of all patients presenting to our Dermatologic Surgery unit who was recorded to have developed a postoperative infection with a positive bacterial wound culture from 2011-2020. In total, 491 patients were identified.

Patients were excluded from the study if no bacterial culture was performed for the corresponding infection, if they had a positive wound culture but no clinical suspicion of postoperative infection, or if they had developed a postoperative infection from a surgery not performed at our surgical unit. Patient demographics, comorbidities, surgical details, antibiotic prophylaxis, culture results and resistances were identified.

All data analysis was performed in SPSS. Chi square analysis was applied for the analysis of categorical variables. A significance level of $\mathrm{p}<0.05$ was applied for final reporting.

\section{Results}

Of the 491 identified patients, 260 patients were included in the analysis. The demographics of our samples are outlined in Table 1 . The average age of patients was 68.6 (SD 13.1) years, with a preponderance of Caucasian (95.0\%) male (66.9\%) cases. The patients had an average of 6.1 (SD 11.3) prior Mohs micrographic surgeries and 2.5 (SD 3.8) prior cutaneous excisions. Almost 50\% of patients were either current smokers or previous smokers. In terms of medical history, 9.3\% of patients had a history of diabetes mellitus, $37.1 \%$ of patients had a prescribed anticoagulant, and $15.4 \%$ were immunosuppressed.

Consistent with prior studies, the majority of infections were of surgical sites on the lower extremities (38.5\%), followed by the upper 
Citation: Chow M, Miller A, Jiang SIB. Anatomic Location is the Best Predictor of Bacterial Species in Postoperative Infections. J Clin Investigat Dermatol. 2021;9(1): 4

Table 1: Demographics and clinical characteristics of patients with postoperative wound infections.

\begin{tabular}{|c|c|c|}
\hline Demographics & Number & $\begin{array}{c}\text { Percent / Standard } \\
\text { deviation }\end{array}$ \\
\hline Age & 68.6 & 13.1 \\
\hline \multicolumn{3}{|l|}{ Ethnicity } \\
\hline Hispanic or Latino & 3 & 1.2 \\
\hline Not Hispanic or Latino & 254 & 97.7 \\
\hline Unknown/not reported & 3 & 1.2 \\
\hline \multicolumn{3}{|l|}{ Race } \\
\hline Asian & 1 & 0.4 \\
\hline Black or African American & 3 & 1.2 \\
\hline White & 247 & 95 \\
\hline More than one race & 7 & 2.7 \\
\hline unknown/not reported & 2 & 0.8 \\
\hline \multicolumn{3}{|l|}{ Gender } \\
\hline Female & 86 & 33.1 \\
\hline Male & 174 & 66.9 \\
\hline \multicolumn{3}{|l|}{ Tobacco use } \\
\hline Current smoker & 18 & 7 \\
\hline Past smoker & 107 & 41.6 \\
\hline Never smoker & 132 & 51.4 \\
\hline History of diabetes mellitus & 24 & 9.3 \\
\hline Anticoagulant use & 96 & 37.1 \\
\hline Immunosuppressed & 40 & 15.4 \\
\hline Prior Mohs & 6.1 & 11.3 \\
\hline Prior Excision & 2.5 & 3.8 \\
\hline \multicolumn{3}{|l|}{ Location } \\
\hline Scalp & 23 & 8.9 \\
\hline Face & 31 & 12.1 \\
\hline Upper extremities, not hands or feet & 45 & 17.5 \\
\hline Lower extremities, not hands or feet & 99 & 38.5 \\
\hline Neck & 8 & 3.1 \\
\hline Chest & 9 & 3.5 \\
\hline Hands & 8 & 3.1 \\
\hline Feet & 2 & 0.8 \\
\hline Abdomen & 2 & 0.8 \\
\hline Back & 30 & 11.7 \\
\hline \multicolumn{3}{|l|}{ Face location } \\
\hline Nose & 7 & 22.6 \\
\hline Ear & 8 & 25.8 \\
\hline Cheek & 7 & 22.6 \\
\hline Lip & 3 & 9.7 \\
\hline Forehead & 6 & 19.4 \\
\hline \multicolumn{3}{|l|}{ Procedure type } \\
\hline Mohs Surgery & 159 & 61.6 \\
\hline Excision & 95 & 36.8 \\
\hline ED\&C & 1 & 0.4 \\
\hline Biopsy & 3 & 1.2 \\
\hline \multicolumn{3}{|l|}{ Repair type } \\
\hline Linear & 173 & 66.5 \\
\hline Flap & 26 & 10 \\
\hline Graft & 40 & 15.4 \\
\hline Second intent & 21 & 8.1 \\
\hline \multicolumn{3}{|l|}{ Superficial suture material } \\
\hline Prolene & 177 & 73.1 \\
\hline Gut & 25 & 10.3 \\
\hline Nylon & 17 & 7 \\
\hline
\end{tabular}

\begin{tabular}{|c|c|c|}
\hline Silk & 3 & 1.2 \\
\hline Vicryl & 1 & 0.4 \\
\hline Monocryl & 19 & 7.9 \\
\hline \multicolumn{3}{|l|}{ Suture technique } \\
\hline Simple running & 126 & 60 \\
\hline Simple interrupted & 33 & 15.7 \\
\hline Vertical mattress & 19 & 9 \\
\hline Subcuticular & 32 & 15.2 \\
\hline $\begin{array}{l}\text { Change in final treatment following } \\
\text { culture }\end{array}$ & 87 & 30 \\
\hline \multicolumn{3}{|l|}{ Empiric treatment } \\
\hline Doxycycline & 146 & 56.2 \\
\hline Cephalexin & 58 & 22.3 \\
\hline Bactrim & 7 & 2.7 \\
\hline Ciprofloxacin & 16 & 6.2 \\
\hline Mupirocin & 5 & 1.9 \\
\hline Bacitracin & 3 & 1.2 \\
\hline No treatment & 21 & 8.1 \\
\hline Clindamycin & 2 & 0.8 \\
\hline Topical gentamicin & 1 & 0.4 \\
\hline Dicloxicillin & 1 & 0.4 \\
\hline \multicolumn{3}{|l|}{ Final treatment } \\
\hline Doxycycline & 103 & 39.8 \\
\hline Cephalexin & 55 & 21.2 \\
\hline Bactrim & 15 & 5.8 \\
\hline Ciprofloxacin & 55 & 21.2 \\
\hline Mupirocin & 6 & 2.3 \\
\hline Bacitracin & 4 & 1.5 \\
\hline No treatment & 11 & 4.2 \\
\hline Clindamycin & 3 & 1.2 \\
\hline Topical gentamicin & 2 & 0.8 \\
\hline Dicloxicillin & 2 & 0.8 \\
\hline Minocycline & 2 & 0.8 \\
\hline Cefpodoxime & 1 & 0.4 \\
\hline
\end{tabular}

extremities (17.5\%) and face (12.1\%). The majority of the patients had undergone Mohs surgery (61.6\%), and most repairs were linear closures (66.5\%). Polypropylene was the most commonly used superficial suture $(73.1 \%)$, and simple running sutures were most frequently used $(60.0 \%)$ among these patients with SSIs. Doxycycline (56.2\%) and cephalexin (22.3\%) were the most commonly prescribed prophylactic antibiotics.

Methicillin-sensitive staphylococcus aureus (MSSA) was the most commonly cultured bacterium at $42.5 \%$ of cultures, followed by pseudomonas aeruginosa (12.4\%) and methicillin-resistant staphylococcus aureus 8.3\%; MRSA; (Table 2). Most of the MSSA cultures were resistant to penicillin $(73.8 \%)$, and smaller percentages were resistant to erythromycin (17.7\%) and clindamycin (12.8\%). Ninety percent of the pseudomonas cultures were sensitive to all common antibiotics tested; 2 cultures (10.0\%) were resistant to aztreonam and 1 culture (3.3\%) was resistant to cefepime. For MRSA, $100 \%$ were resistant to oxacillin but only $92.9 \%$ were resistant to penicillin. Additionally, most cultures were resistant to cefazolin (96.4\%). The culture result changed the recommended antibiotic 30\% of the time.

Next, we investigated the associations of surgical characteristics to culture results. We would like to emphasize that this section does 
Citation: Chow M, Miller A, Jiang SIB. Anatomic Location is the Best Predictor of Bacterial Species in Postoperative Infections. J Clin Investigat Dermatol. 2021;9(1): 4

ISSN: $2373-1044$

Table 2: Microbiology of bacteria cultured from postoperative wound infections.

\begin{tabular}{|c|c|c|}
\hline Microbiology & Frequency & Percent \\
\hline MSSA & 144 & 42.5 \\
\hline Penicillin Resistance & 20 & 95.2 \\
\hline Clindamycin Resisance & 5 & 23.8 \\
\hline Erythromycin Resistance & 17 & 81 \\
\hline Tetracycline Resistance & 8 & 5.7 \\
\hline Minocycline Resistance & 1 & 0.7 \\
\hline Pansensitive & 32 & 22.7 \\
\hline Pseudomonas Aeruginosa & 42 & 12.4 \\
\hline Aztreonam Resistance & 2 & 10 \\
\hline Cefepime Resistance & 1 & 3.3 \\
\hline Pansensitive & 27 & 90 \\
\hline MRSA & 28 & 8.3 \\
\hline Penicillin Resistance & 26 & 92.9 \\
\hline Clindamycin Resisance & 8 & 28.6 \\
\hline Erythromycin Resistance & 23 & 82.1 \\
\hline Tetracycline Resistance & 3 & 10.7 \\
\hline Oxacillin Resistance & 28 & 100 \\
\hline Rifampin Resistance & 1 & 3.6 \\
\hline Bactrim Resistance & 2 & 7.1 \\
\hline Cefazolin resistance & 27 & 96.4 \\
\hline Pansensitive & 0 & 0 \\
\hline Enterobacter Species & 19 & 5.6 \\
\hline Streptococcus species & 16 & 4.7 \\
\hline Staphylococcus epidermidis & 14 & 4.1 \\
\hline Escherichia coli & 14 & 4.1 \\
\hline Proteus Species & 14 & 4.1 \\
\hline Enterococcus Species & 13 & 3.8 \\
\hline Klebsiella Species & 8 & 2.4 \\
\hline Serratia Species & 7 & 2.1 \\
\hline Citrobacter Species & 7 & 2.1 \\
\hline Other Staphylococcus species & 3 & 0.9 \\
\hline Corynebacter Species & 3 & 0.9 \\
\hline Stenotrophomonas Speces & 3 & 0.9 \\
\hline Morganella Species & 2 & 0.6 \\
\hline Yeast & 2 & 0.6 \\
\hline
\end{tabular}

not discuss the rate of infections but rather the differences in the types of bacteria cultured. Not surprisingly, anatomic location was significantly associated with the type of bacteria cultured $p<0.001$. On the lower extremities, MSSA (40.0\%) was most commonly cultured, followed by pseudomonas (20.0\%) and MRSA (10.0\%). Pseudomonas was mainly cultured from the lower extremities and ears. Other gram negative bacteria (E Coli, Enterobacter, Proteus, Klebsiella) were similarly found almost exclusively on the lower extremities. Though the upper and lower extremities and back were commonly represented in our cases, there were relatively few infections on the abdomen and chest. On the back, 28/30 (93.3\%) infections were with MSSA. Similarly, the infections on the scalp, face (excluding ears) and extremities were most commonly with MSSA (Table 2), though the type of bacteria cultured were not significantly associated with face location $(\mathrm{p}=0.053)$.

The type of repair (linear vs flap vs graft vs healing by secondary intention; $\mathrm{p}=0.003)$ and suture material $(\mathrm{p}<0.001)$ was significantly associated with the type of bacteria cultured as well. As we know, the type of repair and suture material is often dictated by the anatomic location, and posthoc analysis supported that anatomic location confounded the bacteria results by type of repair and suture material. Culture results were not associated with procedure type, suture technique, smoking, diabetes, anticoagulant use or immunosuppression status ( $\mathrm{p}>0.05)$.

\section{Discussion}

In summary, of all cases of SSI in the last 9 years at our institution, the majority occurred with surgical sites on the lower extremities repaired with linear closures. There were a large number of male patients with SSIs in our dataset. Overall, there were only a minority of individuals who were immunosuppressed or had a history of diabetes. Our results augment our previous study [6]: in our pool of SSIs on all body sites in addition to the lower extremities, the simple running suture technique was the most common. MSSA, pseudomonas and MRSA infections were the most common bacterial causes of infection. It is worrisome that some of our pseudomonas cultures are resistant to aztreonam and cefepime. It is also worrisome that MRSA is the third most common cause of SSIs in our sample, with resistance to penicillin, cefazolin and erythromycin approaching $100 \%$ and to tetracyclines approaching $10 \%$. Our results show that despite the type of repair and suture material or technique used, the surgical location is the most predictive of the type of bacteria that will grow during a postoperative wound infection. In general, our observations agree with those from other Centers [7].

We believe that some of our culture results differ from those of prior studies due to our practice of prescribing prophylactic antibiotics, usually doxycycline, for lower extremity surgical sites and large flaps on the nose and ears [8]. This may be the reason that, while prior studies have cited MSSA, MRSA followed by pseudomonas as their three most common causes of SSI on the lower extremities, we report pseudomonas as being the second most common cause of SSIs [9]. Though our study was not designed to show whether doxycycline prophylaxis is effective at decreasing the rate of surgical infections, we show that perhaps prophylaxis can at least change the microbiology of infections.

In terms of sensitivities, most Staphylococcus Aureus strains were sensitive to trimethoprim-sulfamethoxazole or doxycycline if it was not sensitive to cephalexin. Additionally, if doxycycline is not effective at treating a patient's lower extremity SSI, gram-negative coverage is most likely needed. We believe that in addition to poor perfusion pressure in the distal limbs, higher tension and complex closures [9], difficulty cleaning the area and washing of genital and gastrointestinal bacteria over the area during showers could also contribute to high infection rates.

There was a statistically significant association between surgical location and the type of bacteria cultured [8]. The location of the wound appears to be more important in determining the culture results than the type of repair or suture material used. Furthermore, suture technique, patient smoking status, immunosuppression, diabetes, age and sex do not appear to be associated with the type of bacteria cultured from postoperative surgical sites with suspected infections.

As discussed in prior articles, we highlight that postoperative wounds collect bacteria that may not be pathogenic, and culture 
Citation: Chow M, Miller A, Jiang SIB. Anatomic Location is the Best Predictor of Bacterial Species in Postoperative Infections. J Clin Investigat Dermatol. 2021;9(1): 4

results must be interpreted with clinical context. This is the reason that we excluded patients with positive culture results but no clinical signs or symptoms of infection and negative culture results despite some clinical signs of infection.

\section{Limitations}

Our results are from a single institution and were collected using a retrospective technique in the small number of patients whose surgeries have been complicated by postoperative infections. Our results are also skewed by our clinical practice of prescribing prophylactic antibiotics in high risk surgical sites.

\section{Conclusion}

In summary, our study confirms findings from prior investigations of postoperative wound infections and suggests that anatomic location may be the most important determinant of the bacteria cultured from a surgical site. In sum, the microbiology of the cultures from SSIs can help clinicians decide which prophylactic and treatment antibiotics are most appropriate for our surgical patients.

\section{References}

1. Futoryan T, Grande D (1995) Postoperative Wound Infection Rates in Dermatologic Surgery. Dermatologic Surg 21: 509-514.
2. Dixon Aj, Dixon Mp, Askew Da Wilkinson D (2006) Prospective Study of Wound Infections in Dermatologic Surgery in the Absence of Prophylactic Antibiotics. Dermatologic Sur 32: 819-827.

3. Nasseri E (2015) Prospective Study of Wound Infections in Mohs Micrographic Surgery Using a Single Set of Instruments. Dermatologic Surg 41: 1

4. Ken KM, Johnson MM, Leitenberger JJ, Neal DE, Etzkorn JR et al. (2020) Postoperative Infections in Dermatologic Surgery. Dermatologic Surg 1294 1299.

5. Barbieri JS, Fix WC, Miller CJ, Sobanko JF, Shin TM, et al. (2019) Variation in Prescribing and Factors Associated With the Use of Prophylactic Antibiotics for Mohs Surgery. Dermatologic Surg. 868-875.

6. Bari O, Eilers RE Jr, Rubin AG JS, Brian Jiang SI (2018) Clinical Characteristics of Lower Extremity Surgical Site Infections in Dermatologic Surgery Based Upon 24-Month Retrospective Review. J Drugs Dermatology 17: 766-771.

7. Dietert JB, Ko J, Hurst EA (2020) Positive Bacterial Culture Results at a Dermatologic Surgery Center. Dermatologic Surg. 46: 773-779.

8. Rosengren H, Heal C, Smith S (2012) An Update on Antibiotic Prophylaxis in Dermatologic Surgery. Curr Dermatol Rep 1: 55-63.

9. SL M, CC O, RK R, PK P (2005) Antibiotic Prophylaxis in Dermatologic Surgery: Updated Guidelines. Dermatol Surg 31: 83-91. 